\title{
Nimbya alternantherae reported for the first time to cause leaf and stem necrosis of Alternanthera philoxeroides (alligatorweed) in Pakistan
}

\author{
Khalid Pervaiz Akhtar ${ }^{1}$, Nighat Sarwar ${ }^{1} \&$ Imran-Ul-Haq $^{2}$ \\ ${ }^{1}$ Nuclear Institute for Agriculture and Biology, Jhang Road, Faisalabad, Pakistan; ${ }^{2}$ Plant Pathology Department, University \\ of Agriculture, Faisalabad, Pakistan
}

Author for correspondence: Khalid P. Akhtar, e-mail: kpervaiz_mbd@yahoo.com

\begin{abstract}
A severe leaf and stem necrosis disease of Alternanthera philoxeroides (alligatorweed) was examined in the summer of 2011. Symptoms on leaves and stems of $A$. philoxeroides consisted of round to oval straw colored spots with maroon margins resulting in chlorosis, severe defoliation and withering of stems. The causal pathogen isolated on V-8 agar medium was morphologically identified as Nimbya alternantherae. Pathogenicity of $N$. alternantherae was proven on healthy A. philoxeroides plants. This is the first report of $A$. philoxeroides necrosis caused by N. alternantherae in Pakistan.
\end{abstract}

Key words: Alternanthera philoxeroides, Nimbya alternantherae, herbaceous weed, identification.

Alternanthera philoxeroides (Mart.) Griseb., also known as alligatorweed, is a herbaceous amphibious weed native to South America. It is a weed species in aquatic and riparian regions of temperate and tropical climates of the world and has spread to North America, Asia and Australia (Barreto \& Torres, 1999; Cox, 2011). It has become a serious problem, forming dense monotypic mats blocking drainage and irrigation channels, interfering with traffic on navigable waterways, and restricting fishing and other uses of freshwater bodies. It can also result in deterioration of aquatic ecosystems leading to reduced levels of oxygen in the water and provides optimal conditions for mosquito breeding (Barreto \& Torres, 1999).

In August 2011, A. philoxeroides plants growing in and around the water channels of experimental fields at Nuclear Institute for Agriculture and Biology (NIAB), Faisalabad, Pakistan, were found to be heavily affected by a leaf and stem necrosis disease. Initial symptoms on leaves started as numerous circular (about $0.1 \mathrm{~mm}$ in diam.) purple to red spots, visible on both adaxial and abaxial leaf surfaces (Figure 1). As the disease progressed, spots expanded and became larger, sunken, often irregular, up to $10 \mathrm{~mm}$ in diam., with grayish-brown necrotic centers and purple to red borders. Sometimes spots coalesced along the mid-rib or originated from margins of the leaf followed by occasional chlorosis and in some cases yellowing of the entire leaf, causing premature abscission (Figure 1) as previously reported by Gilbert et al. (2005). On stems, lesions were relatively rare and elongated, often sunken, purple streaks with a small brown centre.
For the isolation and identification of the involved pathogen, symptomatic A. philoxeroides leaves were collected from 10 plants. These leaves were surface sterilized in $0.25 \%$ sodium hypochlorite solution for $3 \mathrm{~min}$, rinsed 3 times in sterile distilled water and dried on blotter paper under sterile air flow. Small $(5 \times 5 \mathrm{~mm})$ segments of symptomatic leaf tissues were aseptically excised, transferred into Petri plates containing 20\% V-8 juice agar medium and incubated at $25^{\circ} \mathrm{C}$ under a 12 -h light-dark regime.

A grey fluffy fungal growth resulted on which conidiophores and conidia of the pathogen appeared after approximately 5 days. Microscopic investigations revealed that hyphae were smooth, septate, light yellow to straw brown, irregularly branched at right, acute angles, with constrictions at the base of branches, and with club-shaped swellings when old. Conidiophores were semi-macronematous, mononematous, acroauxic, short, with 2-5 cells, erect or ascending without terminal or intercalary nodal swellings, very rarely branched, slightly wider than the vegetative hyphae from which they arise, slightly swollen at the apex, with an apical scar, smooth and pale brown. Conidia were pale brown, 80-115 $\times 18$ $20 \mu \mathrm{m}$ (av. $97.5 \times 19 \mu \mathrm{m}$ ), solitary, acrogenous, obclavate, multicellular with 2-18 cells, arising through a small pore in the conidiogenous apex, straight or slightly curved, usually tapering towards the apex, with basal cell rounded and apical cell slightly rostrate, with 6-10 transverse septa, rarely with two longitudinal septa, often slightly constricted at the septa, smooth or inconspicuously verruculose and bearing a very long and slender beak up to 2.5 times the conidium body length, the beak exhibiting an apical swelling (Figure 2). 


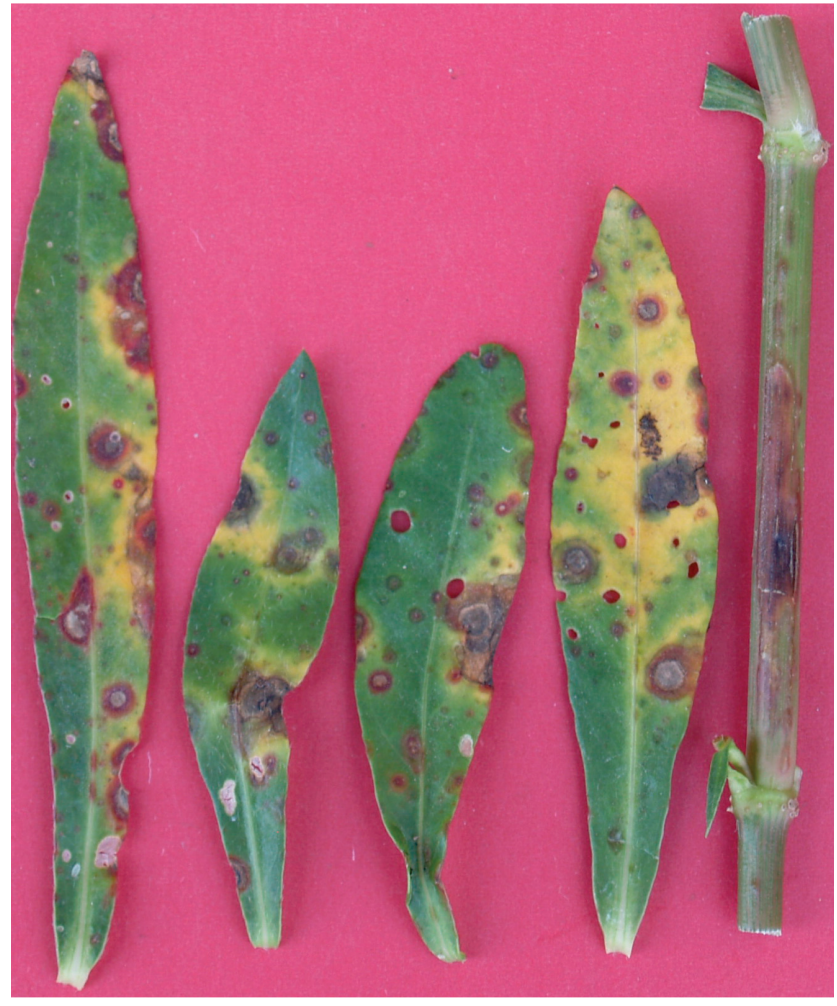

FIGURE 1 - Naturally infected leaves and stem with sunken straw colored spots with maroon borders, few spots coalesced, followed by occasional chlorosis.

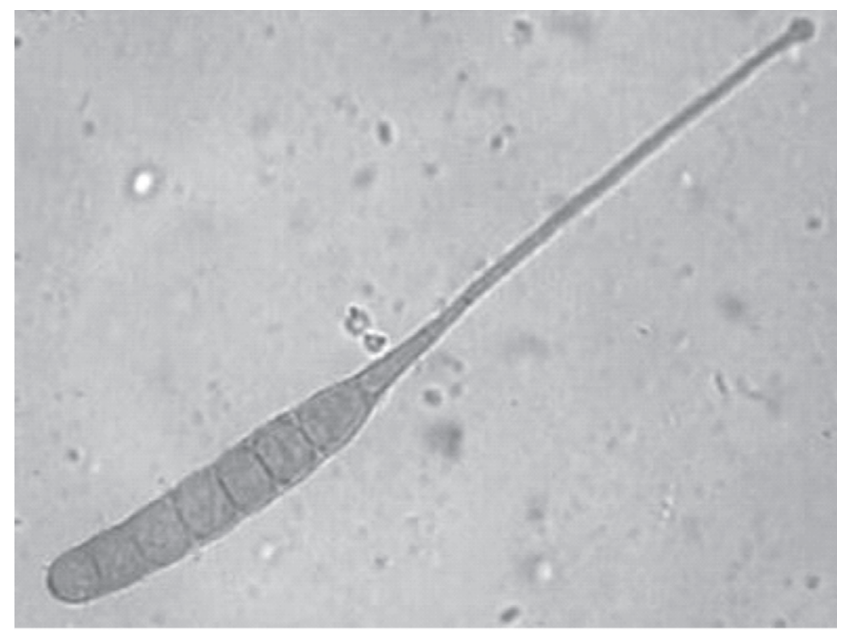

FIGURE 2 - Conidium of Nimbya alternantherae.

These morphological and cultural characteristics of the isolated pathogen coincided with the description of Nimbya alternantherae (Holcomb \& Antonop.) E.G. Simmons \& Alcorn (Holcomb \& Antonopoulos, 1976; Simmons, 1995), therefore the pathogen was identified as $N$. alternantherae. Nimbya was segregated from Alternaria (Simmons, 1989) based on morphology and cultural characteristics. Nimbya alternantherae was originally described as Alternaria alternantherae Holcomb \& Antonop., a pathogen causing leaf spot disease of $A$. philoxeroides (Holcomb \& Antonopoulos, 1976; Holcomb, 1978). A representative specimen of $N$. alternantherae from A. philoxeroides was deposited in the Herbarium First Fungal Culture Bank of Pakistan (FCBP), Institute of Agricultural Sciences (IAGS), P.U., Lahore, on living leaves of A. philoxeroides with Accession No. of FCBP\# 1291.

To confirm the pathogenicity of the obtained isolate, the protocol of Gilbert et al. (2006) was followed. A wild isolate of $N$. alternantherae was multiplied on V-8 agar medium under 12-h light dark cycle. After 10 days $15 \mathrm{~mL}$ of sterilized distilled water were added to each plate and conidia from fungal colonies were carefully loosened with a glass slide. Conidial suspensions were adjusted to $10^{5}$ conidia $/ \mathrm{mL}$ and one drop of Tween 20 surfactant per litter of the conidial suspension was added. Twenty $A$. philoxeroides leaves were excised from healthy glasshouse grown plants, washed several times in sterilized distilled water and blotted dry. These leaves were immersed in the conidial suspensions for 2-3 min and placed in sterile Petri plates (two leaves per plate) on a bed of three filter papers pre-wetted with $3 \mathrm{~mL}$ of sterilized distilled water (Figure 3) (Gilbert et al., 2006). Leaves were incubated in an incubator at $25-30^{\circ} \mathrm{C}$, with a $16 \mathrm{hr}$ light and $8 \mathrm{hr}$ dark regime. A similar set treated with distilled water only was included as a negative control.

Three to five days after inoculation, numerous pinpoint maroon spots were observed on the inoculated leaves. After 7-9 days these spots expanded causing complete leaf chlorosis similar to the symptoms examined under field condition. Nimbya alternantherae with similar cultural and morphological characteristics was re-isolated consistently from these leaves. However, leaves treated with distilled water (negative control) remained green for up to 15-17 days.

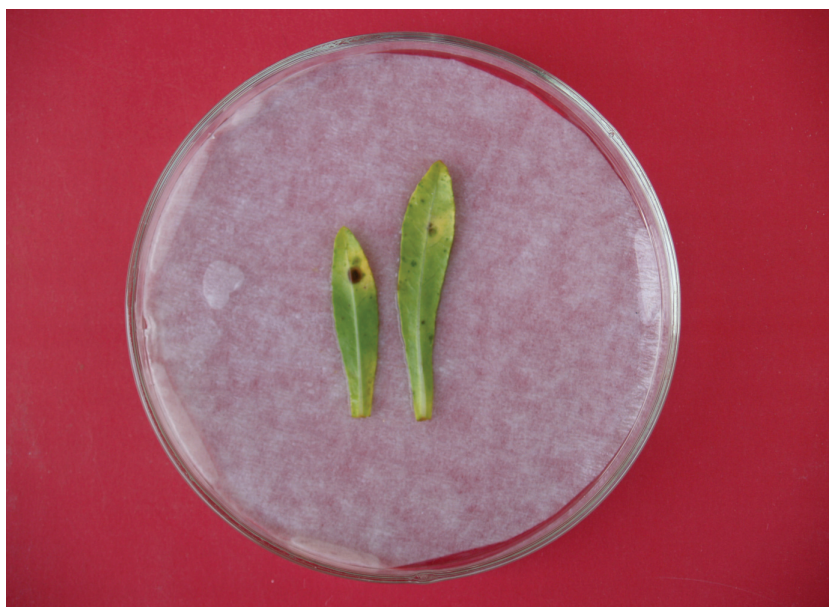

FIGURE 3 - Leaves with pinpoint round to oval maroon spots after artificial inoculation with $N$. alternantherae. 
This is the first report of $N$. alternantherae as a causal pathogen of leaf and stem necrosis of $A$. philoxeroides in Pakistan. $N$. alternantherae naturally causes severe infection on A. philoxeroides producing leaf spots resulting in severe defoliation and occasionally death. Nimbya alternantherae has previously been reported to cause similar disease on $A$. philoxeroides in South America (Barreto \& Torres, 1999), USA (Holcomb \& Antonopoulos, 1976) and China (Xiang et al., 1998). However, from Australia a morphologically different isolate of Nimbia was found to be associated with A. philoxeroides causing similar disease symptoms (Gilbert et al., 2005). Pathogenicity and host range studies performed previously suggest that $N$. alternantherae is pathogenic to three members of Amaranthaceae species (A. philoxeroides, Celosia cristata L., and C. plumose Hort.ex Burvenich), two of Chenpodiaceae (Beta vulgaris L. and Spinocia oleracea L.) and one of the Portulacaceae (Portulaca halimoides L.) (Pomella et al., 2007). This fungus thus has potential as a useful biological control agent of $A$. philoxeroides (Gilbert et al., 2005; Demuner et al., 2006; Cox, 2011). However, intensive studies are still needed on the environmental impact and application technology on the efficiency of $N$. alternantherae as a mycoherbicide for the problems caused by this weed.

\section{ACKNOWLEDGEMENTS}

The authors thank the Pakistan Atomic Energy Commission for providing the financial support. We are also thankful to Mr. Muhammad Tanvir Elahi, (RA), NIAB, for his useful assistance.

\section{REFERENCES}

Barreto RW, Torres Andre NL (1999) Nimbya alternantherae and Cercospora alternantherae: two new records of fungal pathogens on Alternanthera philoxeroides (alligatorweed) in Brazil. Australasian Plant Pathology 28:103-107.

Cox MC (2011) Distribution and management of invasive plant species in the Ross Barnett reservoir. MSc Thesis, Mississippi State University. Starkville MS, USA.

Demuner AJ, Barbosa LCA, Veiga TAM, Barreto RW, King-Diaz B, Lotina-Hennsen B (2006) Phytotoxic constituents from Nimbya alternantherae. Biochemical Systematics and Ecology 34:790795 .

Gilbert RL, Auld BA, Hennecke BR (2005) Leaf and stem spot of Alternanthera philoxeroides (alligatorweed) in Australia caused by Nimbya sp. Plant Pathology 54:585.

Gilbert RL, Gurr GM, Priest MJ, Neilsen S (2006). Evaluation of plant disease for control of alligator weed in Australia. In: $15^{\text {th }}$ Australian Weeds Conference, Proceedings. Adelaide Australia. CAWS. pp. 588-590.

Holcomb GE (1978) Alternaria alternantherae from alligatorweed also is pathogenic on ornamental Amaranthaceae species. Phytopathology 68:265-266.

Holcomb GE, Antonopoulos AA (1976) Alternaria alternantherae: A new species found on alligatorweed. Mycologia 68:1125-1129.

Pomella AWV, Barreto RW, Charudattan R (2007) Nimbya alternantherae a potential biocontrol agent for alligatorweed, Alternanthera philoxeroides. BioControl 52:271-288.

Simmons EG (1989) Macrospora Fuckel (Pleosporales) and related anamorphs. Sydowia 41:314-329.

Simmons EG (1995) Alternaria themes and variations (112-144). Mycotaxon 55:55-163.

Xiang M, Liu R, Yeng Y (1998) Nimbya alternantherae, a new record of the genus Nimbya from China. Mycosystema 17:283.

TPP 569 - Received 2 April 2012 - Accepted 14 September 2012 Section Editor: Alan Wood 